\title{
AS INFECÇÕES SEXUALMENTE TRANSMISSÍVEIS NA PERSPECTIVA DE ADOLESCENTES NA PRÉ-PUBERDADE
}

\author{
Beatriz Moreira Furtado,' Silvia Piedade de Moraes, ${ }^{2}$ José Roberto da Silva Brêtas ${ }^{3}$ \\ SEXUALLY TRANSMISSIBLE INFECTIONS IN THE PERSPECTIVE OF ADOLESCENTS \\ IN PRE-PUBERTY
}

\section{LAS INFECCIONES SEXUALMENTE TRANSMISIBLES EN LA PERSPECTIVA DE ADOLESCENTES EN LA PRÉ-PUBERDAD}

\begin{abstract}
Resumo: Estudo qualitativo realizado com um grupo de adolescentes na pré-puberdade entre I 0 e II anos de idade, com objetivo de verificar o conhecimento sobre as Infecções Sexualmente Transmissíveis (IST) e identificar os meios que utilizam para a obtenção de informações sobre o tema. Os dados foram obtidos por meio de oficinas e analisados pelo método de análise de conteúdo do tipo categorial. Desvelaram-se as categorias: conceito de IST; as IST conhecidas; transmissão, prevenção e tratamento; fontes de informação; dúvidas sobre IST. Concluiu-se que é necessário melhorar o conhecimento e a autocrítica das ações educativas relacionadas à sexualidade, abordando essa temática conceitual antes das IST. Apesar da idade, os participantes demonstraram ter informações sobre IST. Os principais meios de comunicação utilizados para obtenção de informações sobre o tema foram: internet; televisão; pais; professores; e colegas de escola. Concluiu-se que o grupo adolescente na pré-puberdade é o mais propício para o início de intervenções primárias sobre sexualidade e IST.
\end{abstract}

Palavras-chave: Adolescente. Educação em saúde. Educação sexual. Infecções sexualmente transmissíveis.

\begin{abstract}
A qualitative study was carried out with a group of pre-pubescent adolescents between 10 and II years old in order to verify their knowledge of Sexually Transmitted Infections (STI) and to identify the means that they use to obtain information about the subject. Data was obtained through workshops and analysed using the content analysis method and organised according to categorical type. The following categories were used: STI concept; known STI; transmission, prevention, and treatment; information sources; doubts about STI. It was concluded that it is necessary to improve the knowledge and self-criticism of educational actions related to sexuality, addressing this conceptual theme before STIs. Despite the age, the participants demonstrated to have information about STI. The principal means of communication used to obtain information on the subject were: the internet, television, parents, teachers, and schoolmates. We concluded that the pre-pubescent adolescent group is most favourably placed to receive the initiation of primary interventions concerning sexuality and STI.
\end{abstract}

Keywords: Adolescent. Health education. Sex education. Sexually transmitted infections.

Resumen: Estudio cualitativo realizado con un grupo de adolescentes prepúberes de entre I0 y I I años, con el objetivo de verificar conocimientos sobre Infecciones de Transmisión Sexual (ITS) e identificar los medios que utilizan para obtener información sobre el tema. Los datos se obtuvieron a través de talleres y se analizaron mediante el método de análisis de contenido categórico. Se dieron a conocer las categorías: concepto de CTI; las ITS conocidas; transmisión, prevención y tratamiento; fuentes de información; dudas sobre IST. Se concluyó que es necesario mejorar el conocimiento y la autocrítica de las acciones educativas relacionadas con la sexualidad, abordando este tema conceptual antes de las ITS. A pesar de la edad, los participantes demostraron tener información sobre las ITS. Los principales medios de comunicación utilizados para obtener información sobre el tema fueron: internet; televisión; padres maestros y

\footnotetext{
Enfermeira. Universidade Federal de São Paulo (Unifesp). E-mail: beatrizmfurtado@gmail.com

2 Pedagoga. Prof ${ }^{a}$ Dr $^{a}$ da Universidade de Guarulhos (UNG). Especialista em Sexualidade Humana na área de Educação Sexual (Sbrash). E-mail: silviapmoraes@hotmail.com

3 Psicólogo. Professor Orientador do Programa de Pós-Graduação em Educação e Saúde na Infância e na Adolescência da Universidade Federal de São Paulo (Unifesp). Especialista em Sexualidade Humana na área de Educação Sexual (Sbrash). E-mail: jrsbretas@gmail.com
} 
compañeros de escuela. Se concluyó que el grupo de adolescentes en prepubertad es el más propicio para el inicio de intervenciones primarias sobre sexualidad e ITS.

Palabras clave: Adolescente. Educación en salud. Educación sexual. Infecciones de transmisión sexual.

\section{Introdução}

No presente estudo, adotamos a orientação do Departamento de Vigilância, Prevenção e Controle das Infecções Sexualmente Transmissíveis (IST), do Vírus da Imunodeficiência Humana (HIV) ou Síndrome da imunodeficiência adquirida (Aids) e das hepatites virais que passaram a usar a nomenclatura "IST" no lugar de Doenças Sexualmente Transmissíveis (DST). A nova denominação é uma das atualizações da estrutura regimental do Ministério da Saúde por meio do pelo Decreto n 8.901/2016 publicada no Diário Oficial da União em II de novembro de 2016, Seção I, páginas 3 a I7. Porém, mantivemos o termo "DST", nas narrativas dos(as) participantes devido à sua incorporação na linguagem do senso comum.

Para a Organização Mundial da Saúde (OMS), o Fundo de População das Nações Unidas e o UNICEF (1989), a adolescência é representada por indivíduos na faixa etária entre 10 e 19 anos. No Brasil, o Estatuto da Criança e Adolescente (ECA) a define na faixa etária de 12 a 18 anos incompletos (BRASIL, 1996). Entretanto, neste estudo, usamos o termo "adolescência" de acordo com a OMS, destacando especificamente a subdivisão biológica da puberdade, ou seja, com sujeitos pré-púberes com idades entre 10 e I I anos e seus conhecimentos sobre IST.

De acordo com o Boletim Epidemiológico de 2019 (BRASIL, 2020), a maior concentração dos casos de Aids no Brasil foi observada entre pessoas jovens com idade entre 25 e 39 anos, em ambos os sexos. Essa concentração também pode indicar que a infecção tenha ocorrido na adolescência já que o período de infecção e detecção pode ocorrer entre seis e 12 anos (MAHAT; SCOLOVENO; SCOLOVENO, 2016). De acordo com os dados do Ministério da Saúde, entre 2008 e 2018, houve redução nas taxas de detecção em indivíduos do sexo masculino até 14 anos e homens de 30 a 59, e entre o sexo feminino houve redução em todas as faixas etárias. Mesmo assim, a taxa de detecção entre homens jovens é quatro vezes maior que mulheres na mesma faixa etária, apontando cristalizada vulnerabilidade entre esse segmento.

Dados tão expressivos entre a população jovem mostram comportamentos de risco, situações de vulnerabilidade social e escassez de conhecimento sobre sexualidade e saúde sexual na adolescência. Estudos de revisão relatam a falta de conhecimento dos adolescentes sobre IST e, mesmo os jovens que possuem maior nível de conhecimento ou até mesmo os que são sexualmente ativos, não têm atitudes distintas mais preventivas em relação ao sexo ou ao comportamento de alto risco (CELESTINE; JOHNSON; BOODRAM, 2016; PHILLIPS; MBIZVO, 2016; SAMKANGE-ZEEB; MIKOLAJCZYK; ZEEB, 20I3).

Essas atitudes inadequadas apresentadas estão atreladas às informações erradas ou incompletas provenientes de fonte duvidosas ou às estratégias de educação frágeis, que trabalham apenas na propagação de informação e não para mudanças reais de comportamento sexual dos jovens (MAHAT; SCOLOVENO; SCOLOVENO, 2016).

A maioria dos estudantes tem informações corretas sobre o HIV/Aids e sabe que se trata de uma IST, porém, poucos sabem sobre outras infecções, como a clamídia e o Vírus do Papiloma Humano (HPV) (DRAGO et al., 2016; SAMKANGE-ZEEB; MIKOLAJCZYK; ZEEB, 20I3; VAIDAKIS et al., 20I7). Em um estudo norte-americano (MAHAT; SCOLOVENO; SCOLOVENO, 2016), quase metade dos participantes não tinha uma compreensão sobre a diferença entre HIV/Aids/Clamídia, também demonstrando confusão sobre as diferentes opções de tratamento e prognósticos, mesmo se tratando de uma amostragem de adolescentes que procurava ativamente os serviços de cuidado de saúde.

Conhecimentos sobre IST entre adolescentes são estudados no mundo inteiro, porém, no Brasil, ainda está aquém do desejado, principalmente entre os pré-púberes (I0-I4 anos). Para a elaboração deste estudo, realizou-se uma revisão integrativa nas bases de dados PubMed, BVS Adolesc, Lilacs e SciELO, usando várias combinações de descritores como "adolescentes", "infecções sexualmente transmissíveis" e "conhecimento", incluindo artigos publicados entre $1^{\circ}$ de janeiro de 2010 a I 5 de abril de 2017. Raros estudos como os de Oliveira Carvalho, Pinto e Santos (2017) sobre conhecimentos em IST conseguiram incluir poucos adolescentes com I3 e 14 anos. Os resultados mostraram apenas uma publicação com a faixa etária de II anos com relação ao entendimento específico da Aids (GONÇALVES et al., 20I3) e o estudo de Robinson e colaboradores (20I7) que abordou toda a adolescência, explicitando também a relutância dos responsáveis em permitir que seus filhos adolescentes (menores de 14 anos) participassem do estudo.

Parte dessa dificuldade está na crença de que os adolescentes nessa idade são imaturos para lidar com informações sobre sexualidade. A política de ética em pesquisa exige que menores de 18 anos, além do Termo de Consentimento Livre e Esclarecido (TCLE), entregue 
também o Termo de Assentimento assinados por seu responsável. Essa exigência pode ser um dificultador para compreender e traçar políticas públicas eficientes de educação em sexualidade para essa fase da adolescência.

Neste estudo, os participantes são adolescentes na faixa etária entre 10 e II anos de idade, denominada pré-puberdade, um período inícial das mudanças corporais, que antecede o processo da puberdade. Nesse contexto, ressalta-se que

Puberdade é um termo predominantemente biológico e compreende fundamentalmente as transformações corporais, a aparição da menstruação na menina e da ejaculação no menino. Ao contrário, com o termo adolescência, nos referimos ao componente psicológico do processo que é constantemente determinado, modificado e influenciado pela sociedade, não excluindo o fato de ser a puberdade também influenciada pelo fator social. Mas, de uma maneira geral, esta diferenciação é válida (KALINA, I979, p. I I).

Winnicott (1993) afirmou que se examinarmos os processos de amadurecimento, veremos que o jovem nessa fase passa a enfrentar importantes mudanças associadas à puberdade, como o desenvolvimento da capacidade sexual e o aparecimento de manifestações sexuais secundárias. 0 modo como o indivíduo enfrenta essas mudanças e lida com as ansiedades decorrentes delas baseia-se, em grande parte, no padrão organizado desde os primeiros tempos da infância, quando houve uma fase similar de rápido crescimento emocional e físico.

Assim, os participantes dessa pesquisa com idades de 10 e II anos encontram-se em fase de crescimento e desenvolvimento, necessitando de avaliações constantes e intervenções precoces que assegurem sua evolução saudável para a cidadania plena. Essa fase é marcada por descobertas, caracterizadas por uma plasticidade e vulnerabilidade da personalidade. Assim, a pré-puberdade é o momento mais oportuno para intervenções, tornando-as mais eficazes, pois esses indivíduos são mais propensos a incorporar informações e transformá-los em conhecimentos e atitudes positivas enquanto percebem o desenvolvimento de sua sexualidade (CELESTINE; JOHNSON; BOODRAM et al., 2016; DRAGO et al., 2016).

Portanto, uma abordagem de educação em sexualidade em diferentes espaços de socialização deve formar não apenas para a saúde e prevenção, mas para uma vivência segura e feliz de sua sexualidade, a partir de informações corretas capazes de formar habilidades, atitudes, conhecimentos e valores para sua vida (DRAGO et al., 2016; ROBINSON et al., 2017; VAIDAKIS et al., 2017). No que tange à prevenção, é preciso formar com estratégias educativas para tomadas de decisão em comportamentos seguros e, para isso, é preciso pensar no papel da escola, da família, das mídias e ambientes tecnológicos colaborativos, dos grupos e lideranças e dos serviços de saúde, sobretudo na atenção primária e na promoção da saúde. Esse conjunto de esforços é extremamente relevante para superar a precariedade de informações sobre IST e a limitação dos programas escolares em abordar o tema dentro de seus currículos. Além disso, segundo a literatura consultada, se os jovens possuírem o conhecimento, informação e motivação sobre o comportamento sexual seguro, eles serão capazes de mudar de atitude e comportamento (MAHAT; SCOLOVENO; SCOLOVENO, 2016; PHILLIPS; MBIZVO, 2016; ROBINSON et al., 20I7).

Dessa forma, consideramos nesta pesquisa a faixa etária dos pré-púberes e suas particularidades pouco estudadas em relação aos seus conhecimentos sobre IST, como tais conhecimentos são obtidos, quais as repercussões dessas informações no conhecimento em relação à prevenção e como deve ser a abordagem primária para melhorar o conhecimento e motivar a prática de atitudes seguras.

\section{Objetivos e Método}

O estudo usou a pesquisa-intervenção com adolescentes na pré-puberdade por meio de ações educativas em escolas parceiras de um projeto de extensão universitário vinculado ao Grupo de Estudos sobre Corporalidade e Promoção da Saúde (Gecopros) da Universidade Federal de São Paulo (Unifesp) com os seguintes objetivos: (I) verificar o conhecimento sobre as IST; e (2) identificar os meios que utilizam para a obtenção de informações sobre o tema.

Trata-se de um estudo descritivo de abordagem qualitativa com análise de conteúdo categorial, que pode ser definida como um conjunto de técnicas de análise de comunicação visando obter, por procedimentos sistemáticos e objetivos de descrição do conteúdo das mensagens, indicadores que permitem a inferência de conhecimentos relativos às condições de produção/recepção das mensagens organizadas em categorias segundo reagrupamentos analógicos (BARDIN, 2016).

O projeto deste estudo foi encaminhado e aprovado pelo Comitê de Ética da Unifesp, sob Certificado de Apresentação de Apreciação Ética (CAAE) $n^{\circ}$ 434269I 5.7.0000.5505, parecer $n^{\circ}$ I.04I .824, atendendo a todos os procedimentos metodológicos norteados pelos padrões estabelecidos pela Resolução $n^{\circ} 466 /$ I2, que trata das normas de pesquisa envolvendo seres humanos. $O$ estudo foi desenvolvido com $2 \mathrm{I}$ adolescentes de ambos os sexos entre 10 e II anos de idade, que frequentavam o $5^{\mathrm{a}}$ ano do ensino fundamental de uma escola estadual da zona sul do município de São Paulo que entregaram os Termos de Consentimento e Assentimento devidamente assinados. 
A coleta de dados foi realizada na forma de oficinas ${ }^{4}$ em sala de aula com quatro grupos com cinco alunos e um grupo com seis alunos, para registrar o modo que percebiam as IST e, para isso, foram fornecidos: cartolina branca; revista; lápis; canetinha; giz de cera; tesoura e cola. A oficina envolveu a montagem de um painel utilizando recorte-colagem e desenho para responder as seguintes questões

${ }^{4}$ A prática das oficinas consiste precisamente na prática do ofício de pensar sobre a vida e senti-la em vista de pequenas e grandes transformações. A vivência da oficina implica um esforço pedagógico pessoal e coletivo, com a racionalidade e a objetividade próprias da pedagogia, associada à abordagem da dimensão afetiva-emocional da pessoa, de modo a permitir a desconstrução de preconceitos e tabus e reconstrução social dos valores, das crenças sociais e historicamente construídos. Efetivamente, o grupo se torna, por algumas horas, a possibilidade real de experimentação de novos padrões de relacionamento, de problematização dos papéis sociais e de relativização das identidades monolíticas. É esta intencionalidade pedagógica e este olhar psicossocial que nos permitem afirmar que as oficinas se constituem em intervenção psicossocial (RENA, 2006). norteadoras: "O que são IST?”; “Quais são as conhecidas?”; "Onde você obtém informações sobre as IST?".

Após a coleta dos dados, realizou-se a análise do material obtido nos dois momentos da pesquisa, o material individual das questões e o coletado junto à oficina, utilizando a leitura integral das narrativas para compreender suas percepções. O material foi lido de tal forma a obter-se um sentido geral do todo e de cada relato em particular. Em seguida, com a releitura do material, buscou-se o significado para os participantes da temática abordada pelo estudo. Assim, os significados foram agrupados por suas semelhanças, dando origem às categorias a partir da análise das descrições das narrativas (BARDIN, 2016).

\section{Resultados}

Com a estruturação em cinco categorias organizadas e apresentadas no Quadro I, desvelou-se o sistema de significação que, no seu conjunto, revelaram a percepção e o conhecimento dos participantes durante as oficinas coletivas sobre IST.

Quadro I - Distribuição das categorias relacionadas às narrativas produzidas durante as oficinas coletivas

\begin{tabular}{|c|c|}
\hline Categorias & Narrativas dos participantes \\
\hline O conceito de IST & $\begin{array}{l}\text { - DST é uma doença que é transmitida pelo sexo. A gente desenhou uma doença, um vírus, } \\
\text { uma relação sexual; } \\
\text { - DST é sinônimo de doença transmitida pela relação sexual. }\end{array}$ \\
\hline As IST conhecidas & $\begin{array}{l}\text { - Dia } I^{\circ} \text { de dezembro é o dia contra Aids; } \\
\text { - As DST conhecidas são Aids, gonorreia e HPV; } \\
\text { - A gonorreia é uma diarreia que pode ser tratada com ajuda de um médico. Aqui a gente } \\
\text { desenhou a gonorreia e o HPV; } \\
\text { - Aids, gonorreia, tricazonas, HPV e a sífilis; } \\
\text { - Os vírus podem causar a morte, as pessoas que têm Aids têm mais vírus. Esse vírus pega } \\
\text { pelo sexo. Só pelo sexo. A gente só conhece essa Aids. }\end{array}$ \\
\hline $\begin{array}{l}\text { Transmissão, } \\
\text { prevenção e } \\
\text { tratamento }\end{array}$ & $\begin{array}{l}\text { - A prevenção contra a Aids se dá através da camisinha Ollaâ morango; } \\
\text { - A cura do HPV é uma seringa com remédio (vacina) e o coquetel de remédios [desenham } \\
\text { a bebida alcóolica (cocktail)]; } \\
\text { - É eterna [...]. Pega pela relação sexual e pelo sangue. Se você beber água com a mesma } \\
\text { pessoa que tem Aids. A Aids se previne com camisinha; } \\
\text { - A AIDS é uma doença que é transmitida pelo sexo também, no cartaz, a gente desenhou } \\
\text { uma ferida, aí para se prevenir tem que usar a camisinha ou pílulas. O coquetel previne a } \\
\text { Aids também; } \\
\text { - Transmissível pela relação sexual e, para se proteger, é preciso usar a camisinha masculina } \\
\text { e feminina, mas nós desenhamos só a masculina; } \\
\text { - Pode causar a morte, as pessoas que têm Aids têm mais vírus. Pega pelo sexo. No } \\
\text { hospital, podemos detectar as doenças por um simples teste; } \\
\text { - É transmitido pelo beijo, se você tocar na pessoa. É se você se cortou, aí vai e encosta na } \\
\text { ferida dele; } \\
\text { - A gonorreia é uma diarreia que pode ser tratada com ajuda de um médico. E aqui a gente } \\
\text { desenhou a gonorreia; } \\
\text { - HPV é só menina. Acho que é só menina que pega. Só menina! Daí, elas têm que tomar. E } \\
\text { transmite pelo sexo! Para prevenir da HPV, vacina a partir dos I0 até os I } 2 \text { anos; } \\
\text { - O que previne Aids são as vacinas, a camisinha e a pílula; } \\
\text { - Todas as doenças dão para prevenir, menos a Aids. Todas as doenças têm cura até a Aids; }\end{array}$ \\
\hline
\end{tabular}




\begin{tabular}{|c|c|}
\hline Categorias & Narrativas dos participantes \\
\hline $\begin{array}{l}\text { Transmissão, } \\
\text { prevenção e } \\
\text { tratamento }\end{array}$ & $\begin{array}{l}\text { - A prevenção de todas é com camisinha pílula. Aqui é assim, a gente desenhou uma } \\
\text { bactéria que pode ser transmitida pelo sexo ou outros contatos, só que uma bactéria é } \\
\text { muito pequena, aí nós desenhamos uma lupa; } \\
\text { - Uma pessoa com Aids toma mais de } 12 \text { remédios por dia. Porque cada um desses } \\
\text { remédios tem uma função para prevenir; } \\
\text { - É que DST é doença sexualmente transmissível então se o homem tem a doença, que é } \\
\text { sexualmente transmissível, e a mulher transa com ele, ela vai ficar com essa doença; } \\
\text { - Para se prevenir, use preservativos, existem pílulas e coquetel. Aqui, a gente desenhou um } \\
\text { hospital. A figura em cima desenhada é um hospital, você pode buscar ajuda para a DST; } \\
\text { - Desenhamos um vírus, a figura retrata a Aids, gonorreia e o HPV. Desenhamos um } \\
\text { preservativo e aqui tem uma mulher e um homem sem roupas, é uma relação sexual. }\end{array}$ \\
\hline $\begin{array}{l}\text { Fontes de } \\
\text { informação sobre } \\
\text { IST }\end{array}$ & $\begin{array}{l}\text { - A informação vem da internet e televisão. Os pais não. A gente pergunta para a } \\
\text { professora; } \\
\text { - Pela TV, internet, livros, jornais. Com a professora. Às vezes, até com os alunos, com a } \\
\text { minha mãe eu também converso; } \\
\text { - Eu sei tudo pelo meu pai e pela minha mãe; } \\
\text { - A internet e a TV, amigos aqui do colégio. Pais? Os meus, não; } \\
\text { - Na escola, os amigos que sabem conversam comigo. }\end{array}$ \\
\hline Dúvidas sobre IST & $\begin{array}{l}\text { - Quais são as outras doenças? } \\
\text { - O que causa câncer no útero? } \\
\text { - Quais são as curas das doenças: Aids, HPV e gonorreia? } \\
\text { - Se eu beijar alguém que tem AIDS, posso pegar? }\end{array}$ \\
\hline
\end{tabular}

Fonte: Dados da pesquisa.

\section{Discussão}

A primeira associação estabelecida entre os participantes diz respeito ao significado da sigla IST e a relação sexual, mostrando que estão familiarizados com o tema.

Os participantes têm a noção de que a transmissão das IST, no geral, acontece por seres microscópicos, porém confundem os vetores vírus, bactéria, fungos e outros. Além do tipo de transmissor, apresentam o conhecimento de que o ser humano é o hospedeiro definitivo e que por meio da relação sexual se dá a transmissão de um ser contaminado para o não contaminado, bem como por meio do sangue, cortes e machucados. Entretanto, fazem confusão com o modo de transmissão de outras doenças e infecções, quando citam que uma pessoa pode ser contaminada por meio de um copo, assim como constatado na literatura científica (REIS et al., 20I I).

Os achados deste estudo corroboram com estudos promovidos por Mahat, Scoloveno e Scoloveno (2016) e Reis e outros (20I I) de que o conhecimento sobre a prevenção está bastante sedimentado. A maior parte dos participantes associou o tema estudado aos preservativos, principalmente ao masculino, apesar de terem conhecimento do feminino, inclusive fazendo referências a marcas específicas (Ollaâ), o que significa uma grande influência da mídia, como televisão, evidenciada pela reprodução de slogans tanto na fala quanto nos desenhos dos cartazes.
Porém, não têm um conhecimento pleno, pois se referem erroneamente dizendo que a pílula anticoncepcional é um meio de prevenção das IST, fato já verificado em outros estudos (DRAGO et al., 2016; REIS et al., 20II).

A Aids é a única IST conhecida por todos ou a maioria, assim como com os participantes de outros estudos (DRAGO et al., 2016; SAMKANGE-ZEEB; MIKOLAJCZYK, R. T; ZEEB, 20I3). Nesse sentido, muitas vezes, é a única doença conhecida e entendida como um quase sinônimo de IST, sendo citada quatro vezes em um único cartaz e é a primeira fala reproduzida em uníssona pelos grupos quando questionados sobre o que era uma IST: "Aids!". Isso ocorre, provavelmente, pelo fato de que, uma vez em meados de 1980, extensas campanhas de sensibilização sobre esse tópico foram realizadas globalmente dissiminadas e, desde então, vêm sendo amplamente divulgadas pelas mídias.

Os participantes entendem essa patologia como uma doença crônica, evidenciando o fato de não ter cura e relacionando-a ao perigo e à morte. Quanto ao tratamento da Aids, os participantes associaram a ingestão de diversos medicamentos, incluindo o coquetel, porém quando desenham no cartaz o fazem reproduzindo a mistura de bebida alcoólica cocktail. Esse fato demonstra que os participantes reproduzem termos que viram e ouviram, porém, não têm conhecimento do significado, como o exemplo, o coquetel, pois apresentam dificuldade 
na abstração, logo associam ao cocktail, algo que já viram ao menos nos meios de comunicação.

Alguns grupos citaram que o dia $1^{\circ}$ de dezembro é o dia mundial de luta contra a Aids, corroborando novamente para a hipótese de influência da mídia, sendo evidenciado pela narrativa do slogan que aparece na mídia.

Quanto às outras IST, alguns participantes têm ciência da existência da gonorreia, tricomoníase, HPV e sífilis, dados constatados em outros estudos (DRAGO et al., 20I6; SAMKANGE-ZEEB; MIKOLAJCZYK; ZEEB, 20I3), porém não têm nenhum conhecimento específico de outras patologias, verbalizando informações erradas ou genéricas, explicitando diversas vezes que não sabem ao certo tais informações.

Dessas IST, a única que os(as) participantes possuíam algum tipo de informação específica é o HPV, mas apresentavam algumas informações erradas - achavam que somente a mulher seria portadora do vírus do HPV-, e não compreendiam muito bem a transmissão e a prevenção, colocaram em dúvida a existência de remédios ou da vacina, com informação pautada em noticiários na televisão. Atribuímos esse conhecimento a maior atenção da mídia em torno da vacina contra o HPV, priorizando as meninas como o público-alvo da última campanha, assim como apontado em outros trabalhos (DRAGO et al., 2016; SAMKANGE-ZEEB; MIKOLAJCZYK; ZEEB, 20I3; VAIDAKIS et al., 2017).

As fontes de informações mais utilizadas pelos(as) participantes do estudo em forma decrescente foram: internet; televisão; professores/amigos; jornais/livros; e pais/familiares. Dados muito semelhantes a outros estudos (CELESTINE; JOHNSON; BOODRAM, 2016; DRAGO et al., 2016; ROBINSON et al., 20I7; SAMKANGE-ZEEB; MIKOLAJCZYK; ZEEB, 20I3). São dados importantes, pois além da figura do professor, que tem um papel simbólico significativo, esses adolescentes têm acesso a outras fontes - internet, revistas, jornais e livros. Quanto à televisão, é preocupante o valor atribuído à mesma, tratando-se um veículo com tão pouca interatividade. Ainda sobre o mesmo assunto, é importante ressaltar que a família foi pouco citada como fonte de referência o que é muito preocupante, pois a família não deveria estar tão fora do contexto educativo do adolescente. Realidade observada também em alguns estudos (DRAGO et al., 2016; ROBINSON et al., 2017).

Um estudo brasileiro constata que as fontes para obtenção de informação sobre IST dos adolescentes é principalmente a televisão, professores, revistas/jornais/ livros, amigos, família - em ordem decrescente. Ressalta também a baixa referência dos pais e um papel simbólico significativo da figura do professor, o que os levou a inferir a possiblidade de relação entre os meios de comunicação de mídia e a escola (BRÊTAS et al., 2009). As informações sobre transmissão e prevenção não são suficientes para a adoção de comportamentos protetivos, porém, é essa falta de informações básica que aumenta a vulnerabilidade dos adolescentes.

No sentido de acolher e educar, o indivíduo revela ser importante a educação em sexualidade fornecida desde o nascimento pela família e outras instituições de socialização, entre elas a escola. No entanto, a família nem sempre consegue cumprir satisfatoriamente sua função e acaba transferindo-a integralmente a outra instituição de grande influência na formação do sujeito - a escola. Assim, a escola e a família têm papéis complementares na educação em sexualidade (EGYPTO, 2003). A escola deveria complementar com informações corretas e seguras, suprindo lacunas, combatendo preconceitos e desenvolvendo o respeito pelo corpo e sentimentos.

Aescolaé o ambiente social no qual o indivíduo passa grande parte de sua vida, torna-se um cenário apropriado para o desenvolvimento de um programa de educação em sexualidade, como preconizado pela Organização das Nações Unidas para a Educação, a Ciência e a Cultura (Unesco) (20l4), por que além de uma ação direta que exerce sobre os estudantes, indiretamente incentiva a própria família a desempenhar o seu papel. A educação em sexualidade, que na escola deve ser entendida como um processo de intervenção pedagógica, tem como objetivo transmitir informações e problematizar questões relacionadas à sexualidade, incluindo posturas, crenças, tabus e valores a ela associados.

A importância dos meios de comunicação na promoção da saúde e prevenção da doença é bem documentada, uma vez que tanto a exposição de rotina e a utilização estratégica dos meios de comunicação desempenham um papel significativo na promoção da sensibilização, aumento do conhecimento e mudança de comportamentos em saúde (VAIDAKIS et al., 2017).

Em alguns momentos, foi observada a relação que os participantes estabeleciam entre as IST, hospitais e médicos, sendo até dito a frase: "podemos detectar as doenças por um simples teste", como se reproduzissem algum texto de campanha. Eles têm a ideia medicalocêntrica e hospitalocêntrica atualmente transmitida pela mídia e, em nenhum momento, fazem referências às Unidades Básicas de Saúde (UBS) ou aos postos de saúde, equipamentos da saúde primária, responsáveis principalmente pela prevenção em nosso atual sistema de saúde.

Algumas dúvidas foram consideradas comuns entre os grupos como outras IST, a causa e a cura destas, sobre o câncer de colo uterino e o beijo como forma de transmissão da Aids. A exposição das dúvidas mostrou como os participantes interessaram-se pelo tema e, que atividades como oficinas são suficientes para despertar a curiosidade dessa faixa-etária. Os dados monstraram a necessidade e o interesse de conhecer outras doenças, bem como o modo de preveni-las, demonstrando como a "prevenção", 
assunto tão abordado na mídia, influencia o pensamento desses adolescentes.

Em um estudo realizado por Carrión e Blanco (20I2) e outros (CELESTINE; JOHNSON; BOODRAM, 2016; DRAGO et al., 20I6; PHILLIPS; MBIZVO, 2016), os adolescentes estão começando cada vez mais cedo com os jogos, as atividades sexuais e outros comportamentos de exposição ao risco de uma gravidez precoce e não planejada ou uma IST, com o pouco conhecimento aparente que demostram ter, assim, a necessidade de reforçar a educação em sexualidade (CELESTINE; JOHNSON; BOODRAM, 2016; DRAGO et al., 2016; PHILLIPS; MBIZVO, 2016).

Nesse contexto, acreditamos que a educação em sexualidade deve começar desde cedo, com um programa adaptado à realidade que estão vivendo os adolescentes, aberto ao diálogo e para suas mudanças incessantes. Por isso, a educação em sexualidade deve iniciar na pré-puberdade, que é o momento no qual se busca e se tem mais acesso às informações sobre a temática, além de ter muitas questões a ser respondidas e esclarecimentos de informações duvidosas e mal interpretadas.

Os adolescentes precisam adquirir conhecimento sobre as IST o quanto antes, porque muitos desenvolvem comportamentos que envolvem práticas sexuais sem consciência suficiente sobre formas de contágio e tratamentos disponíveis (CELESTINE; JOHNSON; BOODRAM, 2016; ROBINSON et al., 2017; VAIDAKIS et al., 2017).

Um estudo realizado com adolescentes entre II e 12 anos comprovou que jovens que receberam educação sexual na escola tiveram maior conhecimento sobre HIV, uso de camisinha e autoeficácia. Foi uma intervenção baseada na teoria da aprendizagem social e na crença de que uma pessoa tem a capacidade de influenciar seus comportamentos (MAHAT; SCOLOVENO; SCOLOVENO, 2016). Os pesquisadores concluiram que, quatro anos após a intervenção, esses estudantes tinham um conhecimento maior do que aqueles que nunca tiveram educação em sexualidade.

O estudo de Mahat, Scoloveno e Scoloveno (2016) constatou que alunos de I I anos estão aptos a receber educação sexual na escola e que essa matéria terá um benefício tanto quanto ao conhecimento quanto a habilidade dessas crianças no futuro. Outro estudo realizado com adolescentes de diversas faixas etárias concluiu que programas de educação sexual devem ser adptados de acordo com a idade, variando o nível de complexidade e técnicas (VAN DER GEUGTEN et al., 20I5). Logo, alunos de II anos devem aprender educação sexual, entretanto com conteúdos com intensidade e detalhamento diferentes de adolescentes de 15 anos. Assim, esses adolescentes de II e I2 anos também terão conhecimento para diminuir os riscos de adquirir IST ou uma gravidez não planejada.

Devido à televisão e à internet, mídias de fácil acesso para a faixa-etária, muitas informações são difundidas repetidamente e se sedimentam em forma de conhecimento para as crianças e adolescentes, nesse caso, é necessário conversar sobre essas informações de forma crítica e construtiva, a fim de avaliar o que foi apreendido e dirimir interpretações e comportamentos que venham gerar constrangimentos, violência, vulnerabilidade e assimetrias de gênero nos inter-relacionamentos.

\section{Considerações finais}

Apesar da limitação deste estudo, por se tratar de um estudo local, as informações obtidas poderão ser utilizadas como base para vários estudos no futuro, bem como subsídios para reflexões, discussões sobre o assunto e para intervenções primárias.

O estudo mostrou que a educação em sexualidade deve iniciar na pré-puberdade, pois os adolescentes já têm conhecimentos sobre o assunto, embora nem sempre estejam corretos ou sirvam para desenvolver atitudes e habilidades para lidar preventivamente sobre a vida sexual. Também se evidenciou que as questões de gênero e sexualidade devem ser abordadas previamente à IST, devido à emergência dessas temáticas, quando o assunto IST é abordado, e por serem temas intimamente correlacionados.

Como apontado pelos adolescentes, o ambiente escolar e a figura docente são destacadas como fontes seguras para informações acerca da sexualidade e que os mesmos, além de não conversarem sobre 0 assunto na família, tendem a evitá-los, preferindo meios como a escola, amigos e informações que circulam por diferentes mídias.

Dessa forma, é preciso investir todo tipo de esforço para assegurar a educação em sexualidade nas escolas, que seja capaz também de educar os responsáveis, destacando sua importância de atuação em parceria, já que a adolescência é um momento de (re)descoberta e que a sexualidade é construída ao longo da vida, a partir da infância, da história pessoal de cada sujeito, entremeada pelas relações interpessoais estabelecidas entre indivíduo e o ambiente e a cultura no qual vive, em contato com ideologias e visões diferenciadas do mundo.

A cidadania também é vivida pelo conhecimento e domínio do próprio corpo, pois este é o referencial da existência humana, no espaço, no tempo e meio social. É referência da autoestima, da liberdade, da identidade sexual, do prazer sexual e pela vida, pelo respeito ao próprio corpo e pelo corpo do outro com quem se relaciona.

O presente estudo tem grande importância como colaboração para o entendimento de questões relacionadas à sexualidade de adolescentes na pré-puberdade como grupo pouco estudado, mostrando que nessa faixa etária os adolescentes já buscam por informações sobre sexualidade e vida sexual, sendo fundamental a inserção de abordagens educativas para o segmento. 


\section{Referências}

BARDIN, L. Análise de conteúdo. São Paulo: Edições 70, 2016.

BRASIL. Lei n 13.005, de 25 de junho de 20/4. Brasília, DF: Presidência da República, 2014. Disponível em: http://www.planalto.gov.br/ CCIVIL_03/_Ato20I I-20I4/20I4/Lei/LI 3005. htm. Acesso em: 07 jul. 2015.

BRASIL. Ministério da Saúde. Boletim Epidemiológico Secretaria de Vigilância em Saúde. Ministério da Saúde. Número Especial, dez. 2019. Disponível em: http://portalarquivos2. saude.gov.br/images/pdf/2019/setembro/25/ boletim-especial-2 I agol 9-web.pdf. Acesso em: 30 jul. 2020.

BRASIL. Ministério da Saúde. Secretaria de Vigilância em Saúde. Programa Nacional de DST e Aids. Manual de controle das doenças sexualmente transmissíveis. Ministério da Saúde, Secretaria de Vigilância em Saúde, Programa Nacional de DST e Aids. 4. ed. Brasília, DF: Ministério da Saúde, 20I5. p. I40.

BRASIL. Ministério da Saúde. Secretaria executiva de Coordenação da Saúde da criança e do adolescente. Programa Saúde do Adolescente: bases programáticas. Brasília, DF: Ministério da Saúde, 1996.

BRÊTAS, J. R. S. et al. Conhecimentos de adolescentes sobre Doenças Sexualmente Transmissíveis: subsídios para prevenção. Acta Paulista de Enfermagem, São Paulo, v. 22, n. 6, p.786-792, dez. 2009. Disponível em: https:// www.scielo.br/pdf/ape/v22n6/a l 0v22n6.pdf. Acesso em: 03 jul. 2020.

CARRIÓN, J. R. C; BLANCO C. I. T. Conductas sexuales en adolescentes de 12 a 17 años de Andalucía. Gaceta Sanitaria, Barcelona, v. 26, n. 6, p. 519-524, nov./dic. 20I2. Disponível em: http:// scielo.isciii.es/pdf/gs/v26n6/original4.pdf. Acesso em: 22 ago. 2020.

CELESTINE, P. C; JOHNSON, E. J; BOODRAM, C. A. S. Sexual Behavior of Female Adolescents on the Spread of HIV/AIDS and Other STDs in Carriacou. Medicine, Baltimore, v. 95, n. 36, p. e4800, Sept. 2016. Disponível em: https://www.ncbi.nlm.nih.gov/pmc/articles/ PMC50239|3/. Acesso em: 22 ago. 2020.
DRAGO, F. et al. A survey of current knowledge on sexually transmitted diseases and sexual behaviour in italian adolescents. International Journal of Environmental Research and Public Health, Switzerland, v. I3, n. 4 p. 422, Apr. 2016.

EGYPTO, A. C. O projeto de orientação sexual na escola. In: EGYPTO, A. C. (org.) Orientação sexual na escola: um projeto apaixonante. São Paulo: Cortez, 2003. p. |3-3|.

GONÇALVES, $H$. et al. HIV/AIDS transmission knowledge among adolescents aged II years from Southern Brazil. Revista Brasileira de Epidemiologia, v. 16, n. 2, 2013. Disponível em: https://www.scielo.br/pdf/rbepid/v16n2/en_I4I5790X-rbepid-16-02-00420.pdf. Acesso em: I5 ago. 2020.

KALINA, E. Psicoterapia de adolescentes: teoria, técnica e casos clínicos. Rio de Janeiro: Francisco Alves, 1979.

MAHAT, G.; SCOLOVENO, M. A.; SCOLOVENO, R. HIV/AIDS Knowledge, SelfEfficacy for Limiting Sexual Risk Behavior and Parental Monitoring. Journal of Pediatric Nursing, [Orlando], v. 3I, n. I, p. 63-69, Jan./Feb. 2016. Disponível em: https://linkinghub.elsevier.com/ retrieve/pii/S08825963 I 50022 I 3. Acesso em: 30 jul. 2020.

OLIVEIRA CARVALHO; Gardenia Raquel; PINTO, Raydelane Grailea Silva; SANTOS, Márcia Sousa Santos. Conhecimento sobre as infecções sexualmente transmissíveis por estudantes adolescentes de escolas públicas. Adolescência \& Saude, Rio de Janeiro, v. I5, n. I, p. 7-I7, jan./mar. 20I8. Disponível em: http:// www.adolescenciaesaude.com/detalhe_artigo. asp?id=703. Acesso em: 03 jul. 2020.

\section{ORGANIZAÇÃO MUNDIAL DA SAÚDE;} FUNDO DE POPULAÇÃO DAS NAÇÕES UNIDAS; UNICEF. Saúde reprodutiva de adolescentes: uma estratégia para ação. Brasília, DF: Ministério da Saúde, 1989.

PHILLIPS, S. J.; MBIZVO, M. T. Empowering adolescent girls in Sub-Saharan Africa to prevent unintended pregnancy and HIV: a critical research gap. International Journal of Gynecology \& Obstetrics, [New York], v. I32, n. I, p. I-3, Jan. 2016. Disponível em: https://obgyn.onlinelibrary. wiley.com/doi/abs/10.1016/j.ijgo.2015.10.005. Acesso em: 20 jul. 2020. 
REIS, M. et. al. Information and attitudes about HIV/AIDS in portuguese adolescents: State of art and changes in a four-year period. Psicothema, Oviedo, Spain v. 23, n. 2 p. 260-266, Apr. 201 I. Disponível em: http://www.psicothema.com/ PDF/3880.pdf. Acesso em: I0 jul. 2020.

RENA, Luiz Carlos Castello Branco. Educação em saúde: construindo uma pedagogia do cuidado na escola pública. In: I CONGRESSO INTERNACIONAL DE PEDAGOGIA SOCIAL, I., 2006. Proceedings online... Faculdade de Educação, Universidade de São Paulo, Disponível em: http://www.proceedings.scielo.br/scielo. php?script $=$ sci_arttext\&pid $=$ MSC0000000092 $006000100029 \& \operatorname{lng}=e n \& n r m=a b n$. Acesso em: 10 jul. 2020.

ROBINSON, K. H. et al. Responsibilities, tensions and ways forward: parents' perspectives on children's sexuality education. Sex Education, Australia, v. 17, n. 3, p. 333, 2017. Disponível em: https://www.tandfonline.com/doi/full// 0.1080// 46 81811.2017 .1301904 ? scroll $=$ top\&needAccess $=\operatorname{tr}$ ue. Acesso em: I5 jul. 2020.

SAMKANGE-ZEEB, F. N; MIKOLAJCZYK, R. T; ZEEB, H. Awareness and knowledge of sexually transmitted diseases among secondary school students in two German cities. Journal of Community Health, New York, v. 38, n. 2 p. 293-300, Apr. 2013. Disponível em: https://link. springer.com/article/I0.1007/s I0900-0 I2-96 I4-4. Acesso em: 03 jul. 2020.

UNAIDS. Roteiro de Prevenção do HIV até 2020: acelerando a prevenção em HIV para reduzor em $75 \%$ as novas infecções. Disponível em: https:// unaids.org.br/wp-content/uploads/2019/10/2017 Roteiro-Preven\%C3\%A7\%C3\%A3o-2020.pdf. Acesso em: 30 jul. 2020.

UNESCO. Orientações técnicas de educação em sexualidade para o cenário brasileiro: tópicos e objetivos de aprendizagem. Brasília, DF, 2014.

VAIDAKIS, D. et al. Knowledge of Greek Adolescents on Human Papilloma Virus (HPV) and Vaccination: A National Epidemiologic Study. Medicine, Baltimore, v. 96, n. I, p. e5287, Jan. 2017. Disponível em: https://journals. Iww.com/md-journal/Fulltext/2017/01060/ Knowledge_of_Greek_adolescents_on_human_ papilloma.3.aspx. Acesso em: 03 jul. 2020.
VAN DER GEUGTEN, J. et al. Evaluation of a Sexual and Reproductive Health Education Programme: Students' Knowledge, Attitude and Behaviour in Bolgatanga Municipality, Northern Ghana. African journal of reproductive health, Benin City, Edo State, Nigeria, v. 19, n. 3, p. I26-I36, Sept. 20I5. Disponível em: https:// www.ajrh.info/index.php/ajrh/article/view/859. Acesso em: 03 jul. 2020.

WINNICOTT, D. W. Adolescência: transpondo a zona das calmarias. In: WINNICOTT D.W. A família e o desenvolvimento individual. São Paulo: Martins Fontes, 1993.

\section{WORLD HEALTH ORGANIZATION.}

Department of Reproductive Health and Research. Number of People (all ages) living with HIV. Geneva: WHO, 2012.

\section{WORLD HEALTH ORGANIZATION.}

Department of Reproductive Health and Research. Geneva, [ca. 2014]. Disponível em: http://www.who.int/gho/sti/en/. Acesso em: 02 maio 2014. 